\title{
Exploration of lonic Liquids as Soluble Supports for Organic Synthesis. Demonstration with a Suzuki Coupling Reaction
}

\author{
Weishi Miao and Tak Hang Chan* \\ Department of Chemistry, McGill University, Montreal, Quebec \\ Canada H3A 2K6 \\ Email: Tak-Hang.Chan@mcgill.ca
}

\section{Supporting information}

\section{Experimental and Characterization of Compounds:}

General. All reagents were obtained commercially and were used without further purification unless otherwise noted. Microwave assisted reaction was performed in a domestic microwave oven (Panasonic Model NN-S562WF, $1200 \mathrm{~W}, 2450 \mathrm{MHz}) .{ }^{1} \mathrm{H}$ NMR and ${ }^{13} \mathrm{C}$ NMR spectra were recorded on Varian Mercury-300 (300 MHz) or Mercury-400 (400 MHz) at $20{ }^{\circ} \mathrm{C}$. Chemical shifts for protons are reported in parts per million and referenced to residual protium in deuterated chloroform $(\delta 7.26)$, deuterium oxide $(\delta 4.60)$ or acetone $(\delta 2.06)$. Carbon chemical shifts are reported in parts per million relative to the carbon resonance of the methyl group of chloroform$d_{1}(\delta 77.0)$ or acetone- $d_{6}(\delta 29.8,206)$. Mass spectroscopic data were obtained at the McGill University Mass Spectrometry Facility.

Proparation of 1-(2-hydroxyethyl)-3-methylimidazolium bromide ${ }^{1}$ : A mixture of 1methylimidazole $(3.28 \mathrm{~g}, 0.04 \mathrm{~mol})$ and 1-bromoethanol $(5.0 \mathrm{~g}, 0.04 \mathrm{~mol})$ in a $25 \mathrm{ml}$ round bottom flask equipped with a drying tube was placed in a $100 \mathrm{ml}$ beaker containing $30 \mathrm{ml}$ of water. The reaction mixture was heated intermittently $(360 \mathrm{~W} 60 \mathrm{~s}+50 \mathrm{~s}+30 \mathrm{~s})$ in the microwave oven and mixed occasionally by swirling between intervals. ${ }^{2}$ The temperature of the water bath was below $70{ }^{\circ} \mathrm{C}$ during the course of the reaction. The pale brown oil solidified on cooling and was washed by ether $(10 \mathrm{ml} \times 3)$ and dried at $70{ }^{\circ} \mathrm{C}$ under vacuum. The product was obtained as a pale yellow

\footnotetext{
${ }^{1}$ Dzyuba, S. V.; Bartsch, R. A. Tetrahedron Lett. 2002, 43, 4657.

${ }^{2}$ Law, M. C.; Wong, K. Y.; Chan, T. H. Green Chem. 2002, 4, 328.
} 
solid (8.2g, 99\%); Mp 81- $85{ }^{\circ} \mathrm{C} ;{ }^{1} \mathrm{H}$ NMR (400 MHz, $\left.\mathrm{D}_{2} \mathrm{O}\right) \delta 8.55$ (s, $\left.1 \mathrm{H}\right), 7.31(\mathrm{~s}, 1 \mathrm{H}), 725$ (s, $1 \mathrm{H}), 4.12(\mathrm{t}, J=4.8 \mathrm{~Hz}, 2 \mathrm{H}), 3.73(\mathrm{t}, J=4.8 \mathrm{~Hz}, 2 \mathrm{H}), 3.71(\mathrm{~s}, 3 \mathrm{H})$.

Proparation of 1-(2-hydroxyethyl)-3-methylimidazolium tetrafluoroborate $6:^{3}$ A mixture of 1-(2-hydroxyethyl)-3-methylimidazolium bromide (8.2g, $0.04 \mathrm{~mol}), \mathrm{NaBF}_{4}(8.8 \mathrm{~g}, 0.08 \mathrm{~mol})$ and dry acetonitrile $(25 \mathrm{ml})$ was heated to reflux with stirring for $25 \mathrm{~h}$ under nitrogen. Upon cooling, the white precipitate was filtered off and washed with acetonitrile $(10 \mathrm{ml} \times 2)$. The organic filtrate was concentrated in vacuum to give liquid product $6(8.0 \mathrm{~g}, 95 \%) ;{ }^{1} \mathrm{H}$ NMR $(400 \mathrm{MHz}$, Acetone$\left.d_{6}\right) \delta 8.99(\mathrm{~s}, 1 \mathrm{H}), 7.73(\mathrm{~s}, 1 \mathrm{H}), 7.67(\mathrm{~s}, 1 \mathrm{H}), 4.43(\mathrm{t}, J=4.4 \mathrm{~Hz}, 2 \mathrm{H}), 4.05(\mathrm{~s}, 3 \mathrm{H}), 3.92(\mathrm{t}, J=4.4$ $\mathrm{Hz}, 2 \mathrm{H})$.

Proparation of 3 -iodobenzoyloxyethyl-(1-methylimidazolium tetrafluoroborate 5 and 9: To a mixture of ionic liquid $6(3.21 \mathrm{~g}, 0.015 \mathrm{~mol})$, para or meta-iodobenzoic acid $(4.96 \mathrm{~g}, 0.020 \mathrm{~mol})$ and dimethylaminopyridine $(0.25 \mathrm{~g}, 0.002 \mathrm{~mol})$ in dry acetonitrile $(80 \mathrm{ml})$ was added dicyclohexylcarbodiimide ( $1 \mathrm{M}$ in $\mathrm{CH}_{2} \mathrm{Cl}_{2}, 20 \mathrm{ml}, 0.020 \mathrm{~mol}$ ). The reaction was stirred vigorously for $36 \mathrm{~h}$ at room temperature under nitrogen. After filtration, the organic phase was concentrated in vacuum. The crude residue was washed firstly with benzene $(20 \mathrm{ml} \times 3)$, then water $(10 \mathrm{ml})$ and dried at $70{ }^{\circ} \mathrm{C}$ under vacuum to give the product. 3-p-Iodobenzoyloxyethyl-(1methylimidazolium tetrafluoroborate 5: Slightly yellow solids $(5.92 \mathrm{~g}, 89 \%), \mathrm{Mp} 113-115^{\circ} \mathrm{C}$; ${ }^{1} \mathrm{H}$ NMR $\left(400 \mathrm{MHz}\right.$, Acetone- $\left.d_{6}\right) \delta 9.18(\mathrm{~s}, 1 \mathrm{H}), 7.91(\mathrm{~d}, J=8.0 \mathrm{~Hz}, 2 \mathrm{H}), 7.90(\mathrm{~s}, 1 \mathrm{H}), 7.79(\mathrm{~d}, J$ $=8.0 \mathrm{~Hz}, 2 \mathrm{H}), 7.73(\mathrm{~s}, 1 \mathrm{H}), 4.86-4.76(\mathrm{~m}, 4 \mathrm{H}), 4.06(\mathrm{~s}, 3 \mathrm{H}) ;{ }^{13} \mathrm{C}$ NMR $\left(100 \mathrm{MHz}\right.$, Acetone- $\left.d_{6}\right) \delta$ 165.6, 138.6 (2C), 137.9, 131.7 (2C), 129.7, 124.6, 123.7, 101.4, 64.1, 49.4, 36.6; HRMS (FAB) $\mathrm{m} / \mathrm{z}$ calcd. for $\mathrm{M}^{+} \mathrm{C}_{16} \mathrm{H}_{30} \mathrm{O}_{2}$ 357.0100, found 357.0100. 3-m-Iodobenzoyloxyethyl-(1methylimidazolium tetrafluoroborate 9: Slightly yellow solids $(5.66 \mathrm{~g}, 85 \%), \mathrm{Mp} 76-79{ }^{\circ} \mathrm{C} ;{ }^{1} \mathrm{H}$ NMR $\left(300 \mathrm{MHz}\right.$, Acetone- $\left.d_{6}\right) \delta 9.17(\mathrm{~s}, 1 \mathrm{H}), 8.28(\mathrm{~s}, 1 \mathrm{H}), 8.05(\mathrm{~d}, J=7.8 \mathrm{~Hz}, 1 \mathrm{H}), 8.01(\mathrm{~d}, J=$ $7.8 \mathrm{~Hz}, 1 \mathrm{H}), 7.89(\mathrm{~s}, 1 \mathrm{H}), 7.72(\mathrm{~s}, 1 \mathrm{H}), 7.33(\mathrm{t}, J=7.5 \mathrm{~Hz}, 1 \mathrm{H}), 4.87-4.77(\mathrm{~m}, 4 \mathrm{H}), 4.06(\mathrm{~s}, 3 \mathrm{H})$; ${ }^{13} \mathrm{C}$ NMR $\left(75 \mathrm{MHz}\right.$, Acetone- $\left.d_{6}\right) \delta 164.3,142.4,138.2,137.5,131.7,131.0,129.2,124.2,123.3$, 93.8, 64.0, 49.0. 36.3; HRMS (FAB) $\mathrm{m} / \mathrm{z}$ calcd. for $\left[2 \mathrm{M}^{+}+\mathrm{A}^{-}\right]^{+} \mathrm{C}_{26} \mathrm{H}_{28} \mathrm{O}_{4} \mathrm{I}_{2} \mathrm{BF}_{4}$ 801.0229, found 801.0224 .

General procedure for the coupling reactions of ionic liquid supported iodobenzoate 5 or 9 with arylboronic acids 2a-e in aqueous media: To a solution of ionic liquid supported iodobenzoate 5 or $9(0.125 \mathrm{mmol})$ in $2 \mathrm{ml}$ of degassed water at $80^{\circ} \mathrm{C}$ under nitrogen was added arylboronic acid $(0.187 \mathrm{mmol}$ or $0.250 \mathrm{mmol}$, see table 1$)$ and $\mathrm{CsF}(0.625 \mathrm{mmol})$ in one portion. The resulting mixture was stirred at this temperature for $15 \mathrm{~min}$ before $\mathrm{Pd}(\mathrm{OAc})_{2}(0.018 \mathrm{mmol})$ was added. The reaction system was then stirred vigorously for $22 \mathrm{~h}$ at $80{ }^{\circ} \mathrm{C}$ under nitrogen atmosphere. The solvent was removed at $55^{\circ} \mathrm{C}$ under reduced pressure. The residue was washed with ether $(15 \mathrm{ml} \times 3)$ and small amount of water $(1 \mathrm{ml})$. After decanting the liquid phase, the crude product 8a-e or 10a-e was dried on vacuum pump, examined by NMR and subjected to the next step of cleavage reaction.

(8a): ${ }^{1} \mathrm{H}$ NMR (400 MHz, Acetone- $\left.d_{6}\right) \delta 9.58(\mathrm{~s}, 1 \mathrm{H}), 8.08(\mathrm{~d}, J=8.4 \mathrm{~Hz}, 2 \mathrm{H}), \quad 7.99-7.98(\mathrm{~m}$, $1 \mathrm{H}), 7.79-7.78(\mathrm{~m}, 1 \mathrm{H}), 7.75(\mathrm{~d}, J=8.4 \mathrm{~Hz}, 2 \mathrm{H}), 7.68(\mathrm{~d}, J=8.8 \mathrm{~Hz}, 2 \mathrm{H}), 7.06(\mathrm{~d}, J=8.8 \mathrm{~Hz}$, $2 \mathrm{H}), 4.95-4.78(\mathrm{~m}, 4 \mathrm{H}), 4.12(\mathrm{~s}, 3 \mathrm{H}), 3.87(\mathrm{~s}, 3 \mathrm{H}) ;{ }^{13} \mathrm{C}$ NMR $\left(75 \mathrm{MHz}\right.$, Acetone- $\left.d_{6}\right) \delta 165.8$, $160.7,145.9,138.0,132.2,130.8$ (2C), 128.8 (2C), 128.0, 127.0 (2C), 124.5, 123.7, 115.1 (2C), $63.8,55.6,49.5,36.7$.

\footnotetext{
${ }^{3}$ Branco, L. C.; Rosa, J. N.; Moura Ramos, J. J.; Afonso, C. A. M. Chem. Eur. J. 2002, 8, 3671.
} 
(8b): ${ }^{1} \mathrm{H}$ NMR (400 MHz, Acetone- $\left.d_{6}\right) \delta 9.59(\mathrm{~s}, 1 \mathrm{H}), 8.10(\mathrm{~d}, J=8.4 \mathrm{~Hz}, 2 \mathrm{H}), 7.99-7.98$ (m, $1 \mathrm{H}), 7.80-7.79(\mathrm{~m}, 1 \mathrm{H}), 7.79(\mathrm{~d}, J=8.4 \mathrm{~Hz}, 2 \mathrm{H}), 7.66(\mathrm{~d}, J=8.8 \mathrm{~Hz}, 2 \mathrm{H}), 7.55(\mathrm{~d}, J=8.8 \mathrm{~Hz}$, 2H), 4.96-4.79 (m, 4H), 4.11 (s, 3H), 1.37 (s, 9H).

(8c): ${ }^{1} \mathrm{H}$ NMR $\left(400 \mathrm{MHz}\right.$, Acetone- $\left.d_{6}\right) \delta 9.82(\mathrm{~s}, 1 \mathrm{H}), 8.06(\mathrm{~d}, J=8.8 \mathrm{~Hz}, 2 \mathrm{H}), 8.04-8.03(\mathrm{~m}$, $1 \mathrm{H}), 7.81-7.80(\mathrm{~m}, 1 \mathrm{H}), 7.77(\mathrm{~d}, J=8.8 \mathrm{~Hz}, 2 \mathrm{H}), 7.61(\mathrm{dd}, J=3.6,0.8 \mathrm{~Hz}, 1 \mathrm{H}), 7.57$ (dd, $J=5.2$, $0.8 \mathrm{~Hz}, 1 \mathrm{H}), 7.10(\mathrm{dd}, J=5.2,3.6 \mathrm{~Hz}, 1 \mathrm{H}), 4.98-4.78$ (m, $4 \mathrm{H}), 4.10(\mathrm{~s}, 3 \mathrm{H})$.

(8d): ${ }^{1} \mathrm{H}$ NMR (400 MHz, Acetone- $\left.d_{6}\right) \delta 9.47(\mathrm{~s}, 1 \mathrm{H}), 8.11(\mathrm{~d}, J=8.8 \mathrm{~Hz}, 2 \mathrm{H}), 7.95-7.94(\mathrm{~m}$, $1 \mathrm{H}), 7.78(\mathrm{~d}, J=8.8 \mathrm{~Hz}, 2 \mathrm{H}), 7.74-7.73(\mathrm{~m}, 1 \mathrm{H}), 7.70(\mathrm{~d}, J=8.0 \mathrm{~Hz}, 2 \mathrm{H}), 7.51-7.47(\mathrm{~m}, 2 \mathrm{H})$, 7.44-7.40 (m, 1H), 4.89-4.75 (m, 4H), $4.03(\mathrm{~s}, 3 \mathrm{H})$.

(8e): ${ }^{1} \mathrm{H}$ NMR $\left(400 \mathrm{MHz}\right.$, Acetone- $\left.d_{6}\right) \delta 9.58(\mathrm{~s}, 1 \mathrm{H}), 8.15(\mathrm{~d}, J=8.8 \mathrm{~Hz}, 2 \mathrm{H}), 8.11(\mathrm{~d}, J=8.8$ $\mathrm{Hz}, 2 \mathrm{H}), 7.99-7.98(\mathrm{~m}, 1 \mathrm{H}), 7.89(\mathrm{~d}, J=8.8 \mathrm{~Hz}, 2 \mathrm{H}), 7.87(\mathrm{~d}, J=8.8 \mathrm{~Hz}, 2 \mathrm{H}), 7.79-7.78(\mathrm{~m}, 1 \mathrm{H})$, 4.94-4.81 (m, 4H), $4.11(\mathrm{~s}, 3 \mathrm{H}), 2.64$ (s, 3H).

(10a): ${ }^{1} \mathrm{H}$ NMR $\left(300 \mathrm{MHz}\right.$, Acetone- $\left.d_{6}\right) \delta 9.80(\mathrm{~s}, 1 \mathrm{H}), 8.17-8.16(\mathrm{~m}, 1 \mathrm{H}), 8.01-8.00(\mathrm{~m}, 1 \mathrm{H})$, 7.98-7.95 (m, 1H), 7.87-7.83 (m, 1H), 7.79-7.78 (m, 1H), $7.63(\mathrm{~d}, J=8.7 \mathrm{~Hz}, 2 \mathrm{H}), 7.57-7.52(\mathrm{~m}$, $1 \mathrm{H}), 7.05(\mathrm{~d}, J=8.7 \mathrm{~Hz}, 2 \mathrm{H}), 4.96-4.78(\mathrm{~m}, 4 \mathrm{H}), 4.04(\mathrm{~s}, 3 \mathrm{H}), 3.83(\mathrm{~s}, 3 \mathrm{H})$.

(10b): ${ }^{1} \mathrm{H}$ NMR $\left(300 \mathrm{MHz}\right.$, Acetone- $\left.d_{6}\right) \delta 9.80(\mathrm{~s}, 1 \mathrm{H}), 8.23-8.22(\mathrm{~m}, 1 \mathrm{H}), 8.03-8.00(\mathrm{~m}, 1 \mathrm{H})$, 8.00-7.99 (m, 1H), 7.92-7.89 (m, 1H), 7.78-7.77 (m, 1H), $7.63(\mathrm{~d}, J=8.7 \mathrm{~Hz}, 2 \mathrm{H}), 7.54(\mathrm{~d}, J=$ $8.7 \mathrm{~Hz}, 2 \mathrm{H}), 7.55-7.52(\mathrm{~m}, 2 \mathrm{H}), 4.98-4.85(\mathrm{~m}, 4 \mathrm{H}), 4.05(\mathrm{~s}, 3 \mathrm{H}), 1.36(\mathrm{~s}, 3 \mathrm{H})$.

(10c): ${ }^{1} \mathrm{H}$ NMR (400 MHz, Acetone- $\left.d_{6}\right) \delta 9.66(\mathrm{~s}, 1 \mathrm{H}), 8.22-8.21(\mathrm{~m}, 1 \mathrm{H}), 8.00-7.99(\mathrm{~m}, 1 \mathrm{H})$, $7.96(\mathrm{~d}, J=1.6 \mathrm{~Hz}, 1 \mathrm{H}), 7.94(\mathrm{~d}, J=1.6 \mathrm{~Hz}, 1 \mathrm{H}), 7.80-7.79(\mathrm{~m}, 1 \mathrm{H}), 7.58-7.54(\mathrm{~m}, 3 \mathrm{H}), 7.17(\mathrm{dd}$, $\mathrm{J}=5.2,3.6 \mathrm{~Hz}, 1 \mathrm{H}), 4.97-4.84(\mathrm{~m}, 4 \mathrm{H}), 4.13(\mathrm{~s}, 3 \mathrm{H})$.

(10d): ${ }^{1} \mathrm{H}$ NMR $\left(400 \mathrm{MHz}\right.$, Acetone- $\left.d_{6}\right) \delta 9.55(\mathrm{~s}, 1 \mathrm{H}), 8.23-8.22(\mathrm{~m}, 1 \mathrm{H}), 8.04-8.02(\mathrm{~m}, 1 \mathrm{H})$, 7.98-7.97 (m, 1H), 7.94-7.92 (m, 1H), 7.76-7.75 (m, 1H), 7.71-7.69 (m, 2H), 7.62-7.58 (m, 1H), 7.52-7.48 (m, 2H), 7.44-7.40 (m, 1H),4.92-4.78 (m, 4H), $4.04(\mathrm{~s}, 3 \mathrm{H})$.

(10e): ${ }^{1} \mathrm{H}$ NMR (400 MHz, Acetone- $\left.d_{6}\right) \delta 9.70(\mathrm{~s}, 1 \mathrm{H}), 8.28-8.27(\mathrm{~m}, 1 \mathrm{H}), 8.10-8.07(\mathrm{~m}, 2 \mathrm{H})$, $8.09(\mathrm{~d}, J=8.8 \mathrm{~Hz}, 2 \mathrm{H}), 8.03-8.02(\mathrm{~m}, 1 \mathrm{H}), 8.00-7.97(\mathrm{~m}, 1 \mathrm{H}), 7.84(\mathrm{~d}, J=8.8 \mathrm{~Hz}, 2 \mathrm{H}), 7.80-$ $7.79(\mathrm{~m}, 1 \mathrm{H}), 7.65-7.61(\mathrm{~m}, 1 \mathrm{H}) 4.95-4.78(\mathrm{~m}, 4 \mathrm{H}), 4.12(\mathrm{~s}, 3 \mathrm{H}), 3.87(\mathrm{~s}, 3 \mathrm{H})$.

General procedure for the cleavage reaction of ionic liquid supported coupling product 8a-e or 10a-e: The crude coupling product 8a-e or 10a-e from the previous step was dissolved in ammonia-saturated methanol $(2 \mathrm{ml})$. The resulting solution was then stirred at room temperature overnight. After removing the solvent under reduced pressure, the residue was extracted with ether $(20 \mathrm{ml} \times 3)$. Concentration of the combined ether extracts under reduced pressure afforded biaryl products 3a-e or 11a-e and the yields ( 2 steps from 5 or 9) were shown in Table 1.

$(3 a)^{4}:{ }^{1} \mathrm{H}$ NMR $\left(300 \mathrm{MHz}, \mathrm{CDCl}_{3}\right) \delta 8.07(\mathrm{~d}, J=8.4 \mathrm{~Hz}, 2 \mathrm{H}), 7.61(\mathrm{~d}, J=8.4 \mathrm{~Hz}, 2 \mathrm{H}), 7.57(\mathrm{~d}$, $J=8.4 \mathrm{~Hz}, 2 \mathrm{H}), 6.99(\mathrm{~d}, J=8.4 \mathrm{~Hz}, 2 \mathrm{H}), 3.94(\mathrm{~s}, 3 \mathrm{H}), 3.87$ (s, 3H).

$(\mathbf{3 b})^{5}:{ }^{1} \mathrm{H}$ NMR $\left(400 \mathrm{MHz}\right.$, Acetone- $\left.d_{6}\right) \delta 8.07(\mathrm{~d}, J=8.8 \mathrm{~Hz}, 2 \mathrm{H}), 7.78(\mathrm{~d}, J=8.8 \mathrm{~Hz}, 2 \mathrm{H}), 7.66$ $(\mathrm{d}, J=8.8 \mathrm{~Hz}, 2 \mathrm{H}), 7.54(\mathrm{~d}, J=8.8 \mathrm{~Hz}, 2 \mathrm{H}), 3.91(\mathrm{~s}, 3 \mathrm{H}), 1.37(\mathrm{~s}, 9 \mathrm{H})$.

\footnotetext{
${ }^{4}$ Zhu, L.; Duquette, J.; Zhang, M. J. Org. Chem. 2003, 68, 3729.
} 
$(3 \mathrm{c})^{6}:{ }^{1} \mathrm{H}$ NMR $\left(400 \mathrm{MHz}, \mathrm{CDCl}_{3}\right) \delta 8.03(\mathrm{~d}, J=8.0 \mathrm{~Hz}, 2 \mathrm{H}), 7.67(\mathrm{~d}, J=8.8 \mathrm{~Hz}, 2 \mathrm{H}), 7.42(\mathrm{dd}$, $J=3.6,0.8 \mathrm{~Hz}, 1 \mathrm{H}), 7.36(\mathrm{dd}, J=5.2,0.8 \mathrm{~Hz}, 1 \mathrm{H}), 7.11(\mathrm{dd}, J=5.2,3.6 \mathrm{~Hz}, 1 \mathrm{H}), 3.90(\mathrm{~s}, 3 \mathrm{H})$.

$(3)^{5}:{ }^{1} \mathrm{H}$ NMR $\left(400 \mathrm{MHz}, \mathrm{CDCl}_{3}\right) \delta 8.09(\mathrm{~d}, J=8.8 \mathrm{~Hz}, 2 \mathrm{H}), 7.81(\mathrm{~d}, J=8.8 \mathrm{~Hz}, 2 \mathrm{H}), 7.73(\mathrm{~d}$, $J=8.8 \mathrm{~Hz}, 2 \mathrm{H}), \quad 7.53-7.50(\mathrm{~m}, 2 \mathrm{H}), 7.44-7.40(\mathrm{~m}, 1 \mathrm{H}), 3.91(\mathrm{~s}, 3 \mathrm{H})$.

$(3 \mathrm{e})^{4}:{ }^{1} \mathrm{H}$ NMR $\left(400 \mathrm{MHz}, \mathrm{CDCl}_{3}\right) \delta 8.13(\mathrm{~d}, J=8.8 \mathrm{~Hz}, 2 \mathrm{H}), 8.05(\mathrm{~d}, J=8.8 \mathrm{~Hz}, 2 \mathrm{H}), 7.72(\mathrm{~d}$, $J=8.8 \mathrm{~Hz}, 2 \mathrm{H}), 7.70(\mathrm{~d}, J=8.8 \mathrm{~Hz}, 2 \mathrm{H}), 3.96(\mathrm{~s}, 3 \mathrm{H}), 2.67$ (s, 3H).

(11a) $)^{7}$ : White solids, Mp. $71-73^{\circ} \mathrm{C}\left(\right.$ lit $\left.^{7} 70-71.5^{\circ} \mathrm{C}\right) ;{ }^{1} \mathrm{H}$ NMR $\left(300 \mathrm{MHz}, \mathrm{CDCl}_{3}\right) \delta$ 8.25-8.24 (m, $1 \mathrm{H}), 7.99-7.96(\mathrm{~m}, 1 \mathrm{H}), 7.76-7.73(\mathrm{~m}, 1 \mathrm{H}), 7.56(\mathrm{~d}, J=8.7 \mathrm{~Hz}, 2 \mathrm{H}), 7.51-7.46(\mathrm{~m}, 1 \mathrm{H}), 6.99(\mathrm{~d}, J$ $=8.7 \mathrm{~Hz}, 2 \mathrm{H}), 3.96(\mathrm{~s}, 3 \mathrm{H}), 3.87(\mathrm{~s}, 3 \mathrm{H})$.

(11b) $)^{5}{ }^{1} \mathrm{H}$ NMR $\left(400 \mathrm{MHz}, \mathrm{CDCl}_{3}\right) \delta 8.29-8.28(\mathrm{~m}, 1 \mathrm{H}), 8.00(\mathrm{~d}, J=8.0 \mathrm{~Hz}, 1 \mathrm{H}), 7.79(\mathrm{~d}, J=$ $8.0 \mathrm{~Hz}, 1 \mathrm{H}), 7.58(\mathrm{~d}, J=8.4 \mathrm{~Hz}, 2 \mathrm{H}), 7.49$ (d, $J=8.4 \mathrm{~Hz}, 2 \mathrm{H}), 3.96$ (s, 3H), 1.39 (s, 9H).

(11c) ${ }^{8}$ : Pale yellow oil (lit. $\left.{ }^{8} \mathrm{Mp} .40-42{ }^{\circ} \mathrm{C}\right) ;{ }^{1} \mathrm{H}$ NMR $\left(300 \mathrm{MHz}, \mathrm{CDCl}_{3}\right) \delta 8.29-8.28(\mathrm{~m}, 1 \mathrm{H})$, 7.96-7.92 (m, 1H), 7.81-7.77 (m, 1H), $7.45(\mathrm{t}, J=7.8 \mathrm{~Hz}, 1 \mathrm{H}), 7.39$ (dd, $J=3.6,1.2 \mathrm{~Hz}, 1 \mathrm{H})$, $7.32(\mathrm{dd}, J=5.1,1.2 \mathrm{~Hz}, 1 \mathrm{H}), 7.10(\mathrm{dd}, J=5.1,3.6 \mathrm{~Hz}, 1 \mathrm{H}), 3.96(\mathrm{~s}, 3 \mathrm{H})$.

(11d) ${ }^{9}:{ }^{1} \mathrm{H}$ NMR $\left(400 \mathrm{MHz}\right.$, Acetone- $\left.d_{6}\right) \delta 8.26-8.25(\mathrm{~m}, 1 \mathrm{H}), 8.02-7.99(\mathrm{~m}, 1 \mathrm{H})$, 7.94-7.91 (m, $1 \mathrm{H}), 7.72-7.69(\mathrm{~m}, 2 \mathrm{H}), 7.61(\mathrm{t}, J=7.4 \mathrm{~Hz}, 1 \mathrm{H}), 7.53-7.51(\mathrm{~m}, 2 \mathrm{H}), 7.43-7.40(\mathrm{~m}, 1 \mathrm{H}), 3.93(\mathrm{~s}$, $3 \mathrm{H})$.

(11e): White crystals; Mp. 98-100 ${ }^{\circ} \mathrm{C} ;{ }^{1} \mathrm{H}$ NMR $\left(400 \mathrm{MHz}, \mathrm{CDCl}_{3}\right) \delta 8.31-8.30(\mathrm{~m}, 1 \mathrm{H}), 8.08-$ $8.05(\mathrm{~m}, 1 \mathrm{H}), 8.05(\mathrm{~d}, J=8.4 \mathrm{~Hz}, 2 \mathrm{H}), 7.83-7.80(\mathrm{~m}, 1 \mathrm{H}), 7.71(\mathrm{~d}, J=8.4 \mathrm{~Hz}, 2 \mathrm{H}), 7.54(\mathrm{t}, J=$ $8.0 \mathrm{~Hz}, 1 \mathrm{H}), 3.96$ (s, 3H), $2.66(\mathrm{~s}, 3 \mathrm{H}) ;{ }^{13} \mathrm{C} \mathrm{NMR}\left(100 \mathrm{MHz}, \mathrm{CDCl}_{3}\right) \delta$ 197.4, 166.6, 144.4, 140.0, 136.0, 131.4, 130.7, 129.1, 129.0, 128.9 (2C), 128.2, 127.1 (2C), 52.4, 26.8; HRMS (EI) m/z calcd. for $\mathrm{C}_{16} \mathrm{H}_{14} \mathrm{O}_{3} \mathrm{M}^{+} 254.0943$, found 254.0940.

General procedure for the coupling reactions of methyl $p$-iodobenzoate 1 with arylboronic acids $2 \mathrm{a}-\mathrm{e}$ in aqueous media: A mixture of methyl $p$-iodobenzoate $\mathbf{1}(0.125 \mathrm{mmol})$, arylboronic acid $2(0.187 \mathrm{mmol})$ and $\mathrm{CsF}(0.625 \mathrm{mmol})$ in $2 \mathrm{ml}$ of degassed water was stirred for $15 \mathrm{~min}$ at $80^{\circ} \mathrm{C}$ under nitrogen before $\mathrm{Pd}(\mathrm{OAc})_{2}(0.018 \mathrm{mmol})$ was added. The reaction mixture was stirred vigorously for $22 \mathrm{~h}$ at $80^{\circ} \mathrm{C}$ under nitrogen atmosphere. After cooling, the resulting mixture was extracted with ether $(15 \mathrm{ml} \times 3)$ and the combined organic layers were dried over $\mathrm{MgSO}_{4}$ and concentrated in vacuo. The crude product was then examined by proton NMR. For the reaction of $p-\mathrm{MeOPhB}(\mathrm{OH})_{2} \mathbf{2 a}$, the crude was further purified by flash column chromatography (silica gel 230-400 mesh, hexane-ethyl acetate gradient) to give the biaryl product 3a (See Table 2) and the homocoupling product $\mathbf{4 a}$ as white crystals. 4, $\mathbf{4}^{\prime}$-dimethoxybiphenyl (4a) ${ }^{10}$ : ${ }^{1} \mathrm{H}$ NMR (400 $\left.\mathrm{MHz}, \mathrm{CDCl}_{3}\right) \delta 7.48(\mathrm{~d}, J=8.8 \mathrm{~Hz}, 4 \mathrm{H}), 6.96(\mathrm{~d}, J=8.8 \mathrm{~Hz}, 4 \mathrm{H}), 3.85(\mathrm{~s}, 6 \mathrm{H})$.

\footnotetext{
${ }^{5}$ Wang, X.; Parlow, J. J.; Porco, J. A. Org. Lett. 2000, 2, 3509.

${ }^{6} \mathrm{Li}$, X.; Abell, C.; Ladlow, M. J. Org. Chem. 2003, 68, 4189.

${ }^{7}$ Dauben, Wm. G.; Tanabe, M. J. Am. Chem. Soc. 1953, 75, 4969.

${ }^{8}$ Benati, Luisa; Tiecco, Marcello; Bollettino Scientifico della Facolta di Chimica Industriale di Bologna, Supplemento. 1966, 24, 225.

${ }^{9}$ Kang, S.-K.; Kim, J.-S. ; Yoon, S.-K.; Lim, K.-H.; Yoon, S.-S. Tetrahedron Lett. 1998, 39, 3011.

${ }^{10}$ The Aldrich Libary of NMR Spectra, Pouchert, C. J. Ed II, Vol 1, 854B.
} 
PTIL-MeOPhB-Suz-C roavage

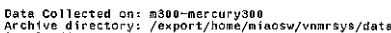

Sample directory

Pulse sequence: s2pul

Solvent : CDC13
Terap. $25.0 \mathrm{C} / 298.1 \mathrm{~K}$

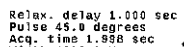

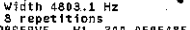

SBSERE H1, 300.0595485 MHE

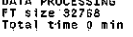

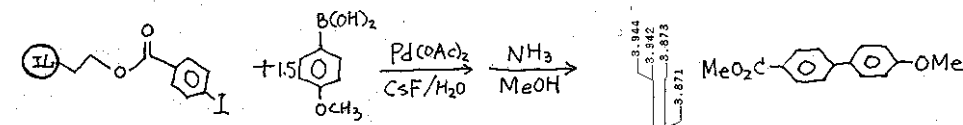

$\underline{5} \quad 2 a$

$\left.\right|^{3}$

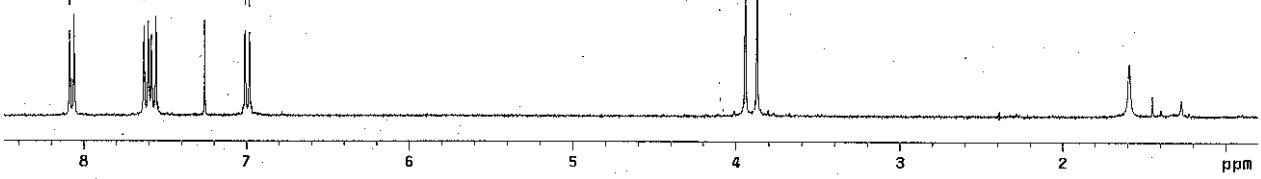

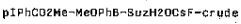

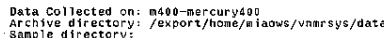

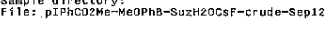

Pulse Sequence: s2pu 1
Solvent: $C 0013$ 3.

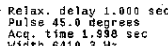

a copetitions

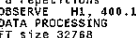

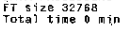
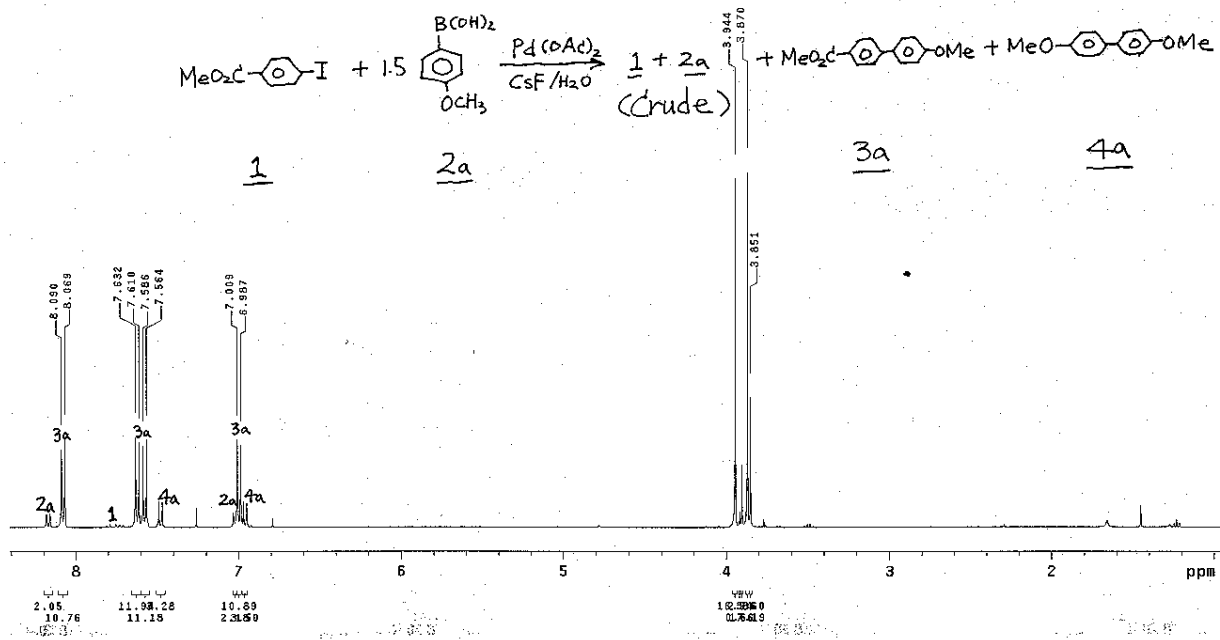